\title{
IMF dependence of the open-closed field line boundary in Saturn's ionosphere, and its relation to the UV auroral oval observed by the Hubble Space Telescope
}

\author{
E. S. Belenkaya ${ }^{1}$, I. I. Alexeev ${ }^{1}$, M. S. Blokhina ${ }^{1}$, S. W. H. Cowley ${ }^{2}$, S. V. Badman ${ }^{2}$, V. V. Kalegaev ${ }^{1}$, and \\ M. S. Grigoryan ${ }^{1}$ \\ ${ }^{1}$ Institute of Nuclear Physics, Moscow State University, Vorob'evy Gory, 119992 Moscow, Russia \\ ${ }^{2}$ Department of Physics \& Astronomy, University of Leicester, Leicester LE1 7RH, UK
}

Received: 27 December 2006 - Revised: 30 April 2007 - Accepted: 7 May 2007 - Published: 4 June 2007

\begin{abstract}
We study the dependence of Saturn's magnetospheric magnetic field structure on the interplanetary magnetic field (IMF), together with the corresponding variations of the open-closed field line boundary in the ionosphere. Specifically we investigate the interval from 8 to 30 January 2004, when UV images of Saturn's southern aurora were obtained by the Hubble Space Telescope (HST), and simultaneous interplanetary measurements were provided by the Cassini spacecraft located near the ecliptic $\sim 0.2$ AU upstream of Saturn and $\sim 0.5 \mathrm{AU}$ off the planet-Sun line towards dawn. Using the paraboloid model of Saturn's magnetosphere, we calculate the magnetospheric magnetic field structure for several values of the IMF vector representative of interplanetary compression regions. Variations in the magnetic structure lead to different shapes and areas of the open field line region in the ionosphere. Comparison with the HST auroral images shows that the area of the computed open flux region is generally comparable to that enclosed by the auroral oval, and sometimes agrees in detail with its poleward boundary, though more typically being displaced by a few degrees in the tailward direction.
\end{abstract}

Keywords. Magnetospheric physics (auroral phenomena; magnetospheric configuration and dynamics; planetary magnetospheres; solar wind-magnetosphere interactions)

\section{Introduction}

The first unambiguous detections of polar ultraviolet (UV) auroras at Saturn were made by the Voyager spacecraft in 1980 and 1981 (e.g. Sandel and Broadfoot, 1981), and were later followed by observations from the Hubble Space Telescope (HST) (Gérard et al., 1995; Trauger et al., 1998). More recent HST observations of higher spatial resolution have

Correspondence to: E. S. Belenkaya

(elena@dec1.sinp.msu.ru) shown that they generally form a ring around each pole $\sim 1^{\circ}-$ $3^{\circ}$ thick, located between $\sim 10^{\circ}-20^{\circ}$ co-latitude (e.g. Cowley et al., 2004a; Gérard et al., 2004; Badman et al., 2006). Such auroral rings can generally be formed either by the solar wind interaction, leading to emissions in the vicinity of the boundary between open and closed field lines, or by the current system associated with the maintenance of magnetospheric plasma corotation deeper within the magnetosphere (e.g. Cowley and Bunce, 2001; Hill, 2001). From an analysis of the latter current system, Cowley and Bunce (2003) concluded that the corotation-enforcement currents at Saturn are too weak and occur at too low a latitude to explain the observed UV auroras, and thus suggested that a solar windrelated origin was the more likely. These results have subsequently been amplified by further modelling work presented by Cowley et al. (2004b), and also by the statistical analysis of the location of the UV auroras presented by Badman et al. (2006), who showed that they occur well poleward of the latitude where the magnetospheric plasma departs from near-rigid corotation according to Voyager data.

Direct evidence of the strong connection between Saturn's UV auroras and the solar wind was first obtained during the joint HST-Cassini campaign in January 2004, when the Cassini spacecraft was upstream of the planet, en route to Saturn orbit insertion (Clarke et al., 2005; Crary et al. 2005; Bunce et al., 2006). Jackman et al. (2004) had shown that the interplanetary medium at the time of the Cassini approach, corresponding to the declining phase of the solar cycle, was strongly structured by corotating interaction regions (CIRs) into a recurrent pattern of high-field compression regions and low-field rarefaction regions. During the few-day compression regions the interplanetary magnetic field (IMF) strength was typically $0.5-2 \mathrm{nT}$, while during the several day rarefaction intervals it was $\sim 0.1 \mathrm{nT}$ or less. This structuring is evident in Fig. 1, where we show the Cassini field and plasma data covering the interval of the HST observations, taken from Badman et al. (2005). The field data were obtained

Published by Copernicus GmbH on behalf of the European Geosciences Union. 


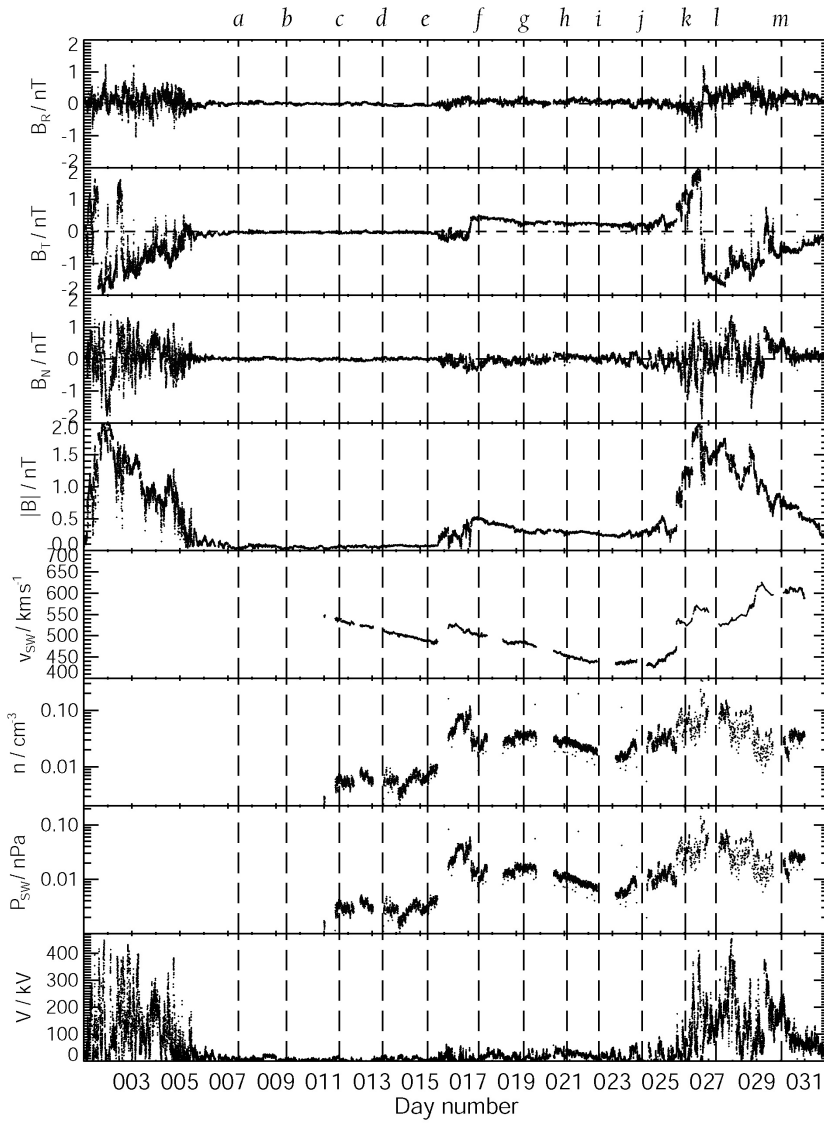

Fig. 1. Stacked plot of Cassini IMF and plasma data obtained during the January 2004 Cassini-HST campaign. The first four panels show the RTN magnetic field components $\left(B_{R}, B_{T}, B_{N}\right)$, and the magnetic field magnitude $|B|$ in $\mathrm{nT}$. The fifth to seventh panels show the solar wind proton velocity $v_{s w}\left(\mathrm{~km} \mathrm{~s}^{-1}\right)$, the solar wind density $n_{p}\left(\mathrm{~cm}^{-3}\right)$, and the dynamic pressure $P_{s w}(\mathrm{nPa})$, respectively. The bottom panel shows the estimated magnetopause reconnection voltage $V(\mathrm{kV})$ using the algorithm of Jackman et al. (2004). The dashed vertical lines indicate the corresponding times of the HST images shown in Fig. 2, adjusted to take into account the solar wind propagation delay and the Saturn-HST light propagation delay (figure adapted from Badman et al., 2005).

by the Cassini magnetic field investigation (Dougherty et al., 2004), and are shown in RTN coordinates. RTN is a right-handed spherical polar system referenced to the Sun's spin axis, with $B_{R}$ directed radially outward from the Sun, $B_{T}$ azimuthal in the direction of planetary motion around the Sun, and $B_{N}$ normal to the other two components, positive northward from the equatorial plane. The plasma parameters were derived from Cassini CAPS particle spectrometer data (Young et al., 2004). The times of the HST imaging intervals are shown by the vertical dashed lines, where account has been taken of the $\sim 17 \mathrm{~h}$ solar wind radial propagation delay from Cassini to Saturn, and of the 68-min light travel time from Saturn to the HST. The solar wind delay is uncertain to within a few hours, however, due to possible non-radial propagation effects and the difference in the heliocentric longitude of Cassini and Saturn (Crary et al., 2005). During this interval Cassini was located near the ecliptic $\sim 0.2 \mathrm{AU}$ upstream of Saturn, and $\sim 0.5 \mathrm{AU}$ off the Sun-planet line toward dawn.

It can be seen that the HST observations encompass two rarefaction regions, the earlier one being somewhat "deeper" than the later, together with two compression regions bounded by interplanetary shocks. The first "minor" compression onset occurred on 16 January, while the second "major" compression onset occurred on 26 January. The auroral observations themselves are shown in Fig. 2, letter coded as in Fig. 1 (Clarke et al., 2005; Bunce et al., 2006). These show the Southern Hemisphere of the planet with the noon meridian toward the top of each image, dawn to the left, and dusk to the right. It can be seen that the auroral distributions observed after the compression onsets in images (f) and (k) (at intervals of $\sim 40$ and $\sim 11 \mathrm{~h}$ after the initial shock, respectively) show contracted brightened ovals, with the whole of the dawn-side polar cap being filled with bright sun-aligned arcs in the latter case. Thus, although the strength of the IMF at Saturn's orbit is typically an order of magnitude less than that at Earth, and the dynamic pressure typically two orders of magnitude less, the interaction with the interplanetary medium nevertheless remains important for Saturn's magnetosphere.

Badman et al. (2005) investigated the variations in the amount of magnetic flux contained within the "dark" region poleward of Saturn's auroras in the January 2004 data shown in Fig. 2, and found that it varied between $\sim 15$ to $\sim 50 \mathrm{GWb}$, with the smallest values being associated with the intervals following compression onsets. On the assumption that the poleward boundary of the auroras represents an approximate proxy for the open-closed field line boundary, in accordance with the above discussion, it was thus inferred that major open flux closure events were triggered by these interplanetary events, with the open flux then increasing again in the subsequent few-day high-field intervals. Belenkaya et al. (2006a) have also studied these data, and in addition to the compression-induced flux closure just mentioned, have also discussed the role of a three-dimensional current system in the auroral dynamics, arising from the solar wind interaction. This current system includes Region 1 field-aligned currents (FACs) concentrated at the open-closed field line boundary, "SBZ" FACs distributed over the polar cap near the cusp for southward IMF (the kronian counterpart of "NBZ" currents at Earth arising under northward IMF conditions), together with the Pedersen currents on open field lines in the ionosphere which close both these systems. The Region 1 currents are added to the ring of upward FAC of comparable magnitude at the boundary of open field lines which is associated with differential plasma rotation between open and closed field lines, such that the overall current system for the 


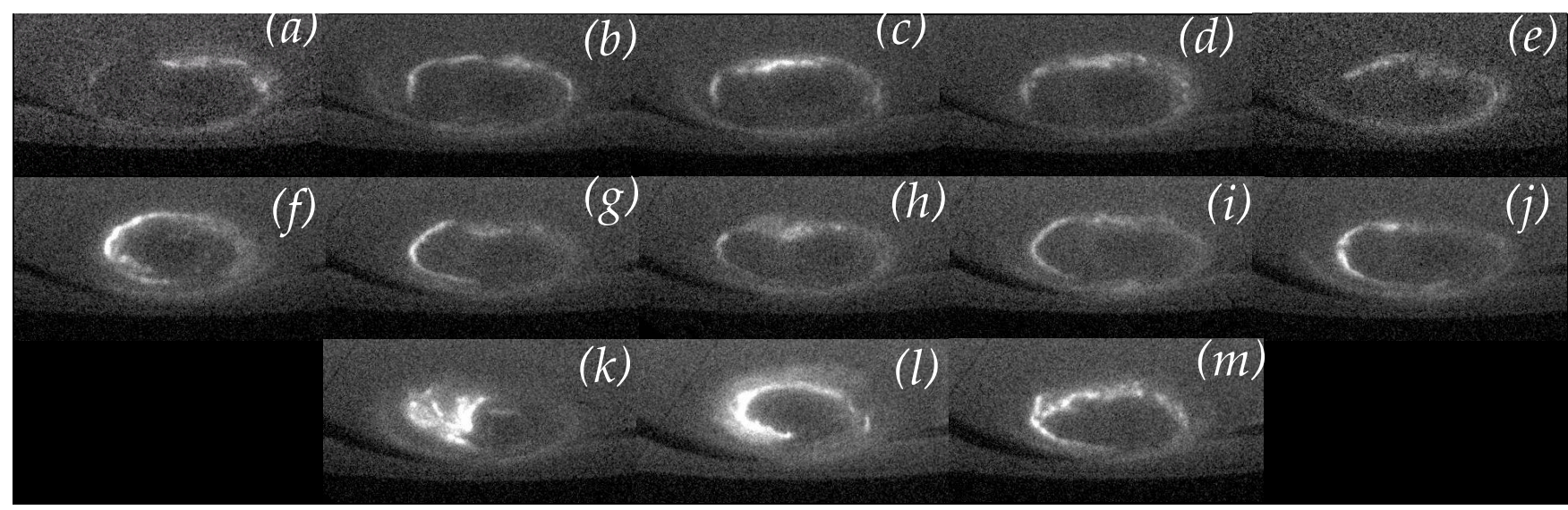

Fig. 2. UV images of Saturn's southern aurora obtained by HST-STIS on 8, 10, 12, 14, 16, 18, 20, 21, 23, 24, 26, 28, and 30 January 2004 (panels a to $\mathbf{m}$, respectively). The panels have been generated by combining individual images obtained on a given HST orbit (Clarke et al., 2005; Bunce et al., 2006). The noon meridian is at the centre top of each plot, with dawn to the left (figure from Bunce et al., 2006).

January 2004 events takes the form of strong upward FAC on the dawn side, with corresponding bright aurora, and weaker upward (or even downward) FAC at dusk, with weaker (or absent) auroras. Figure 2 shows that such dawn-dusk asymmetry is characteristic of the bright active intervals observed in January 2004.

In this paper we investigate IMF influences on the size and position of the open field region at Saturn during these active intervals, corresponding to periods when the interplanetary field was at its strongest, such that the IMF effects may be most evident. Our calculations are based on the Saturn magnetospheric model described by Alexeev et al. (2006) and Belenkaya et al. (2006b), in which the magnetopause is taken to be a paraboloid of revolution about the Saturn-Sun line. Kronian solar-magnetospheric coordinates (KSM) are thus employed, in which the X-axis is directed towards the Sun, Saturn's magnetic moment $\boldsymbol{M}_{S}$ lies in the X-Z plane, and $Y$ completes the right-handed orthogonal triad. It may be noted that for the January 2004 interval the difference between KSM and the heliospheric RTN system employed in Fig. 1 is very small, such that $B_{x} \approx-B_{R}, B_{y} \approx-B_{T}$, and $B_{z} \approx B_{N}$. The main contributors to the model magnetic field are then (i) the intrinsic magnetic (dipole) field of the planet, as well as the shielding magnetopause current which confines the dipole field inside the magnetopause, (ii) the ring current and the shielding magnetopause current that similarly confines its field inside the magnetopause, (iii) the tail currents and their magnetopause closure currents, and (iv) the IMF which penetrates into the magnetosphere. We note that the principal interplanetary influences on the model derive from the solar wind dynamic pressure which determines the position of the magnetopause and hence the overall size of the system, and the direction and strength of the interplanetary field which is reflected in the penetrating IMF component. We also note that the ring current is modelled as a thin equa- torial disc in which the azimuthal current intensity falls as the inverse square of the radial distance, and that the tail current is re-scaled from an earlier terrestrial model.

The parameters which define Saturn's magnetospheric magnetic field in the model are thus as follows: (i) $R_{s s}$ is the distance from Saturn's centre to the subsolar point on the magnetopause; (ii) $R_{r c 1}$ and $R_{r c 2}$ are the distances to the outer and inner edges of the ring current, respectively; (iii) $R_{2}$ is the distance from the planet's centre to the inner edge of the magnetospheric tail current sheet; (iv) the field magnitude of the tail currents at the inner edge of the tail current sheet is $B_{t} / \alpha_{0}$, where $\alpha_{0}=\left(1+2 R_{2} / R_{S S}\right)^{1 / 2}$; (v) $\Psi$ is the tilt angle between the magnetic dipole direction and the KSM $\mathrm{Z}$ axis $\left(\sim 25^{\circ}\right.$ during the January 2004 interval, corresponding to Northern Hemisphere winter conditions); (vi) $B_{r c 1}$ is the radial component of the ring current magnetic field at the outer edge of the ring current; (vii) the effect of the IMF inside the magnetosphere is given by the uniform field $k_{s} \boldsymbol{B}_{\mathrm{IMF}}$, where $\boldsymbol{B}_{\mathrm{IMF}}$ is the IMF vector and $k_{S}$ is the coefficient of its penetration into the magnetosphere. With regard to the latter model assumption, we note that Alexeev (1986) obtained a finite-conductivity solution for the magnetic field in the magnetosheath, in which magnetic field diffusion results in only a partial screening of the IMF by the magnetopause. The magnetic field inside the magnetosphere was then found to be some fraction $k_{S}$ of the external field, depending on the value of the magnetic Reynolds number $R_{m}$. In the next section we calculate the size and location of the open flux region in Saturn's ionosphere using this model, employing parameter values determined by Belenkaya et al. (2006b) appropriate for compressed active conditions. 


\section{Paraboloid model calculations for the cases of $\mathbf{1 6}$ and 26 January 2004}

As can be seen in Fig. 1, during the "minor" compression region occurring between 16 and 18 January, the KSM components of the IMF can be characterised by $\left(B_{x}, B_{y}, B_{z}\right)=(0.0,-0.4,-0.4) \mathrm{nT}$ (Belenkaya et al., 2006a). These values are taken as characteristic of the interval surrounding the maximum in pressure during the "minor" compression, near the "shifted" time of image (f). The solar wind density increased to $0.1 \mathrm{~cm}^{-3}$, while the speed also increased to $\sim 530 \mathrm{~km} \mathrm{~s}^{-1}$. Following this compression, a rarefaction region was then observed between days 19 and 25 , with an IMF field strength $\sim 0.3 \mathrm{nT}$, a density of $0.01-$ $0.04 \mathrm{~cm}^{-3}$, and with almost the same solar wind speed. The "major" compression then occurred on 25 January, and lasted essentially to the end of the interval considered. During this compression the IMF strength was $\sim 1-2 \mathrm{nT}$, the density increased to $\sim 0.03-0.1 \mathrm{~cm}^{-3}$, and the solar wind speed increased to $\sim 630 \mathrm{~km} \mathrm{~s}^{-1}$. Belenkaya et al. (2006a) estimated that during the earlier part of this event the KSM IMF components were approximately $(0.5,-2.0,-1.4) \mathrm{nT}$. These values correspond to the interval near the time of image (k), just prior to the pressure maximum during the compression event. Later in the event, near the time of image (m), the IMF can reasonably be typified by KSM components $(-0.3,0.7,0.7) \mathrm{nT}$. In our calculations below we use the three IMF values given here as representative of the two compression intervals, together with other model parameters intended to reflect the compressed magnetospheric conditions then prevailing. Specifically, we employ the values determined by Belenkaya et al. (2006b) appropriate to the compressed magnetosphere observed during the Pioneer-11 flyby, given by $R_{s s}=17.5 R_{S}, R_{r c 1}=12.5 R_{S}, R_{r c 2}=6.5 R_{S}, R_{2}=14 R_{S}$, $B_{t}=8.7 \mathrm{nT}$, and $B_{r c 1}=3.62 \mathrm{nT}$, where $R_{S}=60330 \mathrm{~km}$ is the equatorial value of Saturn's radius. These values should therefore also provide a reasonable representation for the compressed conditions of interest here, given that the results should not be sensitively dependent on the exact choices.

With regard to the value of the IMF penetration parameter $k_{S}$, we note that Belenkaya (2006a) used values of 0.2 and 0.8 for rough estimations. Earlier, Belenkaya (2004) showed that the value 0.8 was most appropriate at Jupiter for the interpretation of observations of anti-corotational solar winddriven plasma flow in the equatorial magnetosphere. Tsyganenko (2002) found that the best correspondence between his model of the near-Earth magnetosphere and observational data was obtained for an IMF penetration coefficient between 0.15 and 0.8. The larger size of Saturn's magnetosphere compared to Earth (by a factor of $\sim 16$ ) is expected to decrease $k_{S}$ by a factor of $\sim 2$, since $k_{S}$ is proportional to $R_{m}^{-1 / 4}$ and $R_{m}$ is proportional to the size of the magnetosphere. However, as deduced by Alexeev et al. (2003), plasma compression at the bow shock increases $k_{S}$ by a factor of $\sim 2$, and a high level of magnetic field variability in Saturn's magnetosheath can also lead to an increase in $k_{S}$. Overall, we thus expect that $k_{S}$ for Saturn should be similar to or larger than that for Earth. Here we will therefore consider values of $k_{S}$ within the range 0.2 to 0.8 .

In Fig. 3 we present some results illustrating Saturn's magnetic field lines for the above model parameters, together with $k_{S}=0.8$ and KSM IMF vectors given from top to bottom of the figure by $(0.0,-0.4,-0.4),(0.5,-2.0,-1.4)$, and $(-0.3,0.7,0.7) \mathrm{nT}$. The first two are the southwarddirected IMF vectors corresponding to the above "minor" and initial "major" compression regions, while the third is the northward-directed field corresponding to the later part of the "major" compression. The field lines shown start from the ionosphere on the noon and midnight meridians, but, due to the presence of the IMF $B_{y}$ component, are twisted out of the meridian in the region away from the planet. In the left-hand column of Fig. 3 we show the projection of these field lines on the $\mathrm{X}-\mathrm{Z}$ plane, while in the right-hand column we provide an indication of the 3-D structure. In the upper two panels with southward IMF we see that the size of the modelled region of field lines having even one "end" in the ionosphere (open and closed), decreases as the IMF field strength increases. The bottom panel shows that the structure of the model magnetic field lines changes dramatically when the IMF becomes northward-directed.

In Fig. 4 we correspondingly show how the open-closed field line boundary varies in response to the IMF vector, specifically for the Southern Hemisphere, thus matching the auroral images shown in Fig. 2. The view is looking "through" the planet onto the southern pole, with noon on the right side of these figures, and dusk at the top. Panel (a) corresponds to the "minor" compression on 16 January with KSM vector $(0.0,-0.4,-0.4) \mathrm{nT}$ (and $\Psi=25.05^{\circ}$ ). The open field region is displaced to the dusk side of the pole due to the presence of the IMF $B_{y}$ component. In panel (b) the northward $B_{z}$ component has been increased in magnitude to $-1.4 \mathrm{nT}$, such that the KSM components are $(0.0,-0.4,-1.4) \mathrm{nT}$. The open field region shrinks and becomes centred sunward as well as duskward of the pole. Panel (c) shows how the open area responds to the $B_{y}$ field, with an IMF given by KSM components $(0.0,-2.0,-0.4) \mathrm{nT}$. In this case the open region becomes enlarged in area. In panel (d) we then increase the magnitude of the northward field component again to $-1.4 \mathrm{nT}$, so that the KSM components become $(0.0,-2.0,-1.4) \mathrm{nT}$. The increased magnitude of the southward-directed field again results in a decrease in the open area. Panel (e) then shows the effect of changing the $B_{x}$ component, with an IMF vector of $(0.5,-2.0,-1.4) \mathrm{nT}$, which thus now corresponds to the "major" compression region on 26 January (with $\Psi=24.64^{\circ}$ ). The open field region is almost unchanged compared with panel (d) in this case. Finally, in panel (f) we show results for the northward-directed IMF given by $(-0.3,0.7,0.7) \mathrm{nT}$, corresponding to the later part of the "major" compression. In this case the open field line region significantly increases 
$\operatorname{Bimf}=0.0 ;-0.4 ;-0.4 ; \mathrm{ks}=0.8$

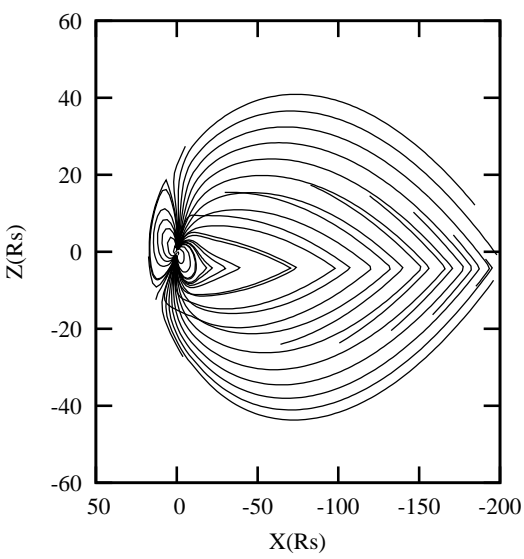

$\operatorname{Bimf}=0.5 ;-2.0 ;-1.4 ; \mathrm{ks}=0.8$

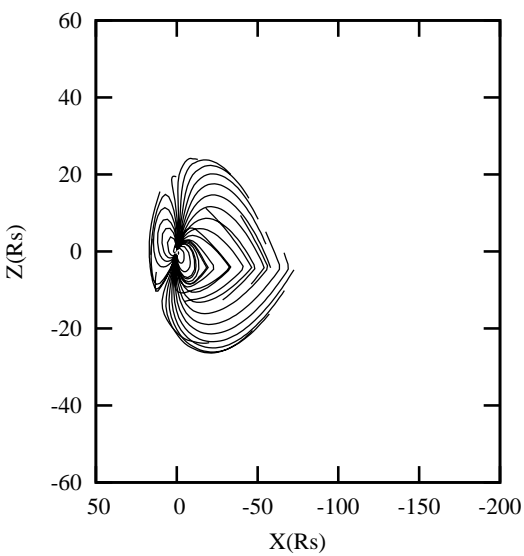

$\operatorname{Bimf}=-0.3 ; 0.7 ; 0.7 ; \mathrm{ks}=0.8$

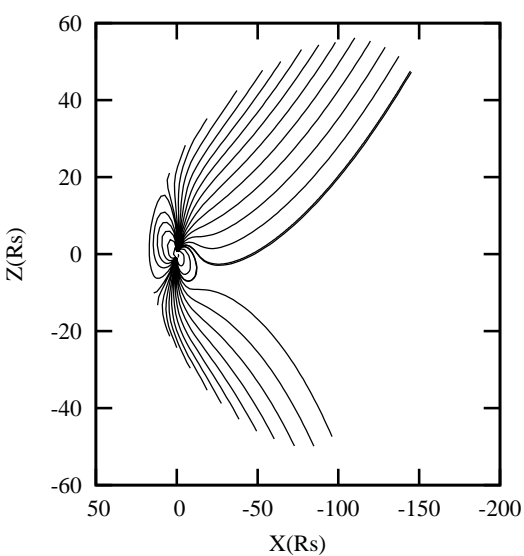

$\operatorname{Bimf}=0.0 ;-0.4 ;-0.4 ; \mathrm{ks}=0.8$

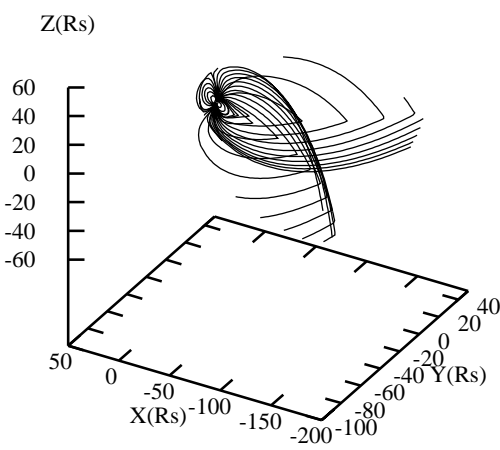

$\operatorname{Bimf}=0.5 ;-2.0 ;-1.4 ; \mathrm{ks}=0.8$

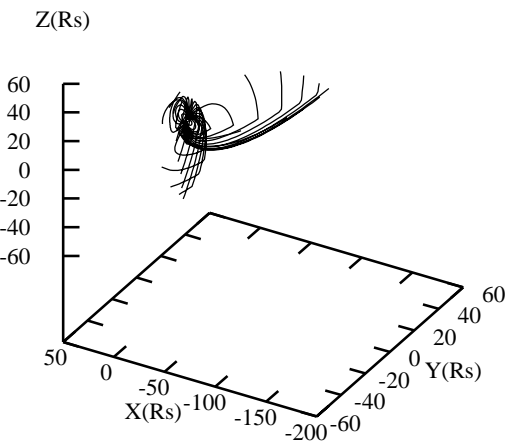

$\operatorname{Bimf}=-0.3 ; 0.7 ; 0.7 ; \mathrm{ks}=0.8$

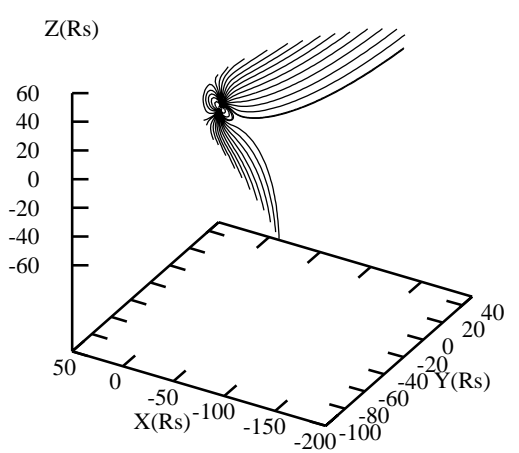

Fig. 3. Plots showing the field lines emerging from Saturn's ionosphere in the noon and midnight meridians. On the left-hand side of the plot the field lines are projected into the X-Z plane, while the 3-D structure is indicated on the right-hand side. The three rows correspond to differing IMF vectors. From top to bottom these are given by KSM components of $(0.0,-0.4,-0.4),(0.5,-2.0,-1.4)$, and $(-0.3,0.7,0.7) \mathrm{nT}$, corresponding to the "minor" field compression interval, and the initial and later "major" compression intervals, respectively. The input model parameters are $R_{S S}=17.5 R_{S}, R_{2}=14 R_{S}, R_{r c 1}=12.5 R_{S}, R_{r c 2}=6.5 R_{S}, B_{r c 1}=3.6 \mathrm{nT}, B_{t}=-8.7 \mathrm{nT}$, and $k_{S}=0.8$. The dipole tilt angle is $\Psi \sim 25^{\circ}$. 
$\operatorname{Bimf}=0.0 ;-0.4 ;-0.4 ; \mathrm{ks}=0.8$

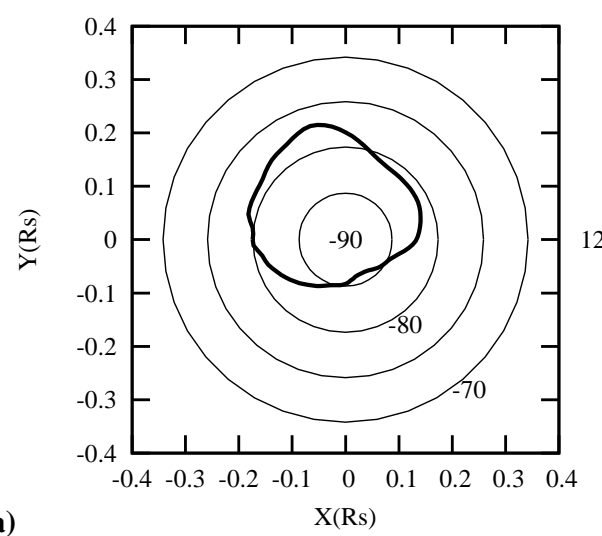

(a)

$\operatorname{Bimf}=0.0 ;-2.0 ;-0.4 ; \mathrm{ks}=0.8$

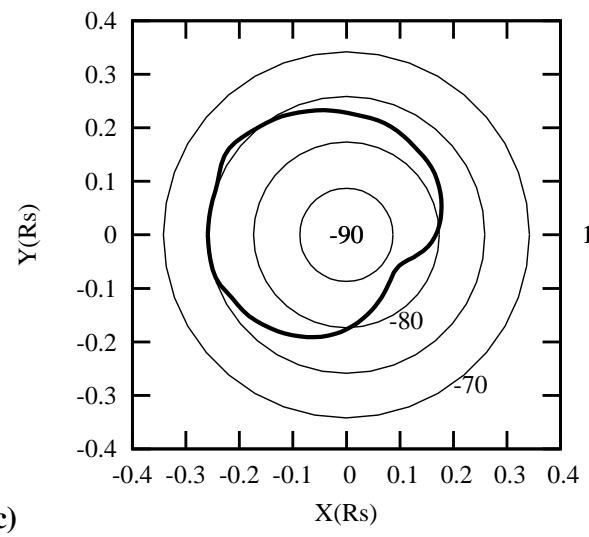

$\operatorname{Bimf}=0.5 ;-2.0 ;-1.4 ; \mathrm{ks}=0.8$

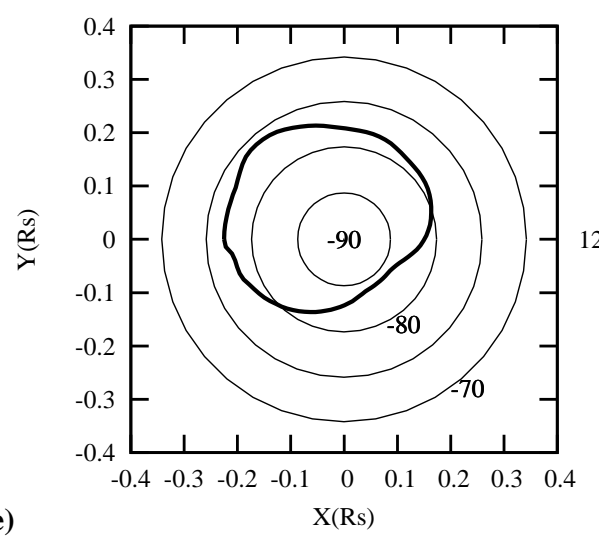

$\operatorname{Bimf}=0.0 ;-0.4 ;-1.4 ; \mathrm{ks}=0.8$

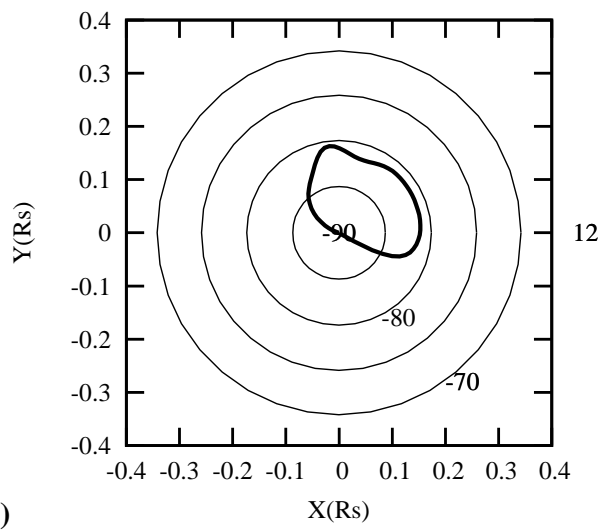

$\operatorname{Bimf}=0.0 ;-2.0 ;-1.4 ; \mathrm{ks}=0.8$

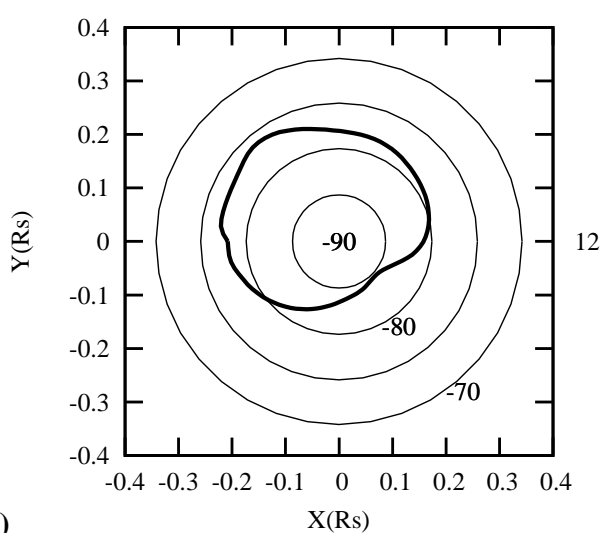

$\operatorname{Bimf}=-0.3 ;+0.7 ;+0.7 ; \mathrm{ks}=0.8$

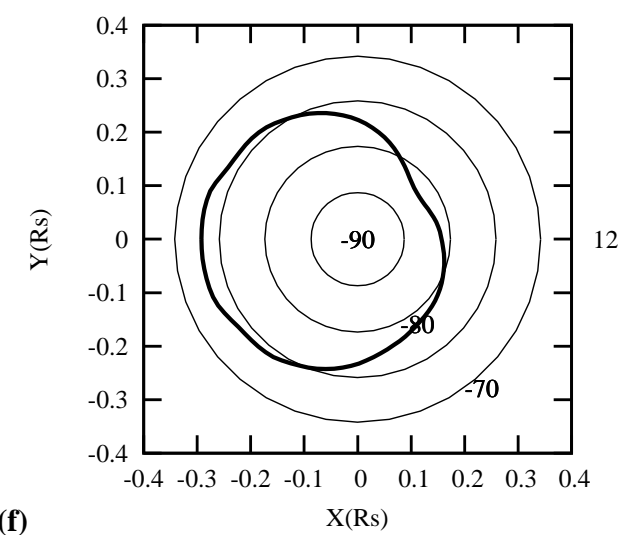

Fig. 4. Open field line regions in Saturn's southern ionosphere calculated using the paraboloid model for various sets of IMF components. In panels (a-f) the KSM components are as follows: (a) $(0.0,-0.4,-0.4) \mathrm{nT}$, (b) $(0.0,-0.4,-1.4) \mathrm{nT},(\mathbf{c})(0.0,-2.0,-0.4) \mathrm{nT},(\mathbf{d})$ $(0.0,-2.0,-1.4) \mathrm{nT}$, (e) $(0.5,-2.0,-1.4) \mathrm{nT}$, and (f) $(-0.3,0.7,0.7) \mathrm{nT}$. The view is looking "through" the planet onto the southern pole, with noon at the right, dusk at the top, and co-latitude is indicated at intervals of $5^{\circ}$. The model parameters are as in Fig. 3.

in size.

In Fig. 5 we also show how the open field line region varies with the value of the IMF penetration coefficient $k_{S}$, again for the Southern Hemisphere. On the left-hand side of the figure we reproduce the results shown in Fig. 4 which correspond to the typical IMF conditions during the "minor" and initial and later "major" compression intervals using $k_{S}=0.8$ (panels a, e, and $\mathrm{f}$ in Fig. 4, respectively). On the right-hand side we show results for the same IMF vector, but with $k_{S}=0.2$. We see that the value of $k_{S}$ smoothly changes the open field line area in the ionosphere. At the same time, the value of $k_{S}$ significantly influences the polar cap potential drop and 
$\operatorname{Bimf}=0.0 ;-0.4 ;-0.4 ; \mathrm{ks}=0.8$

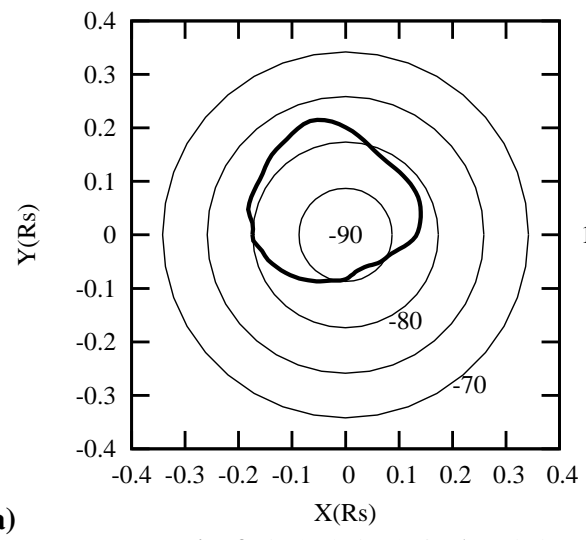

(a)

$\operatorname{Bimf}=0.5 ;-2.0 ;-1.4 ; \mathrm{ks}=0.8$

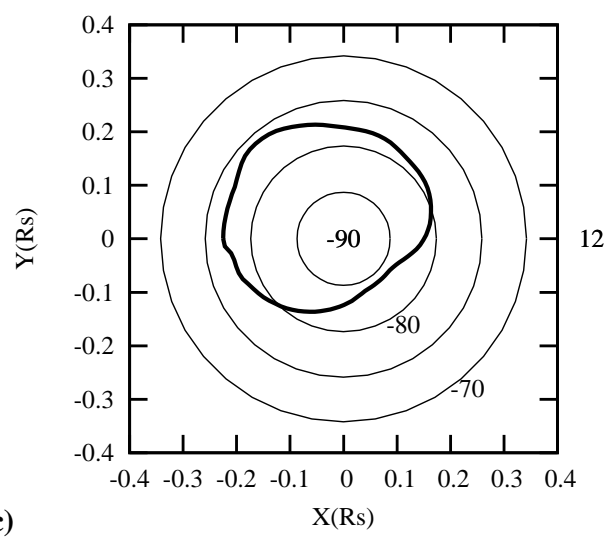

Bimf $=-0.3 ; 0.7 ; 0.7 ; \mathrm{ks}=0.8$

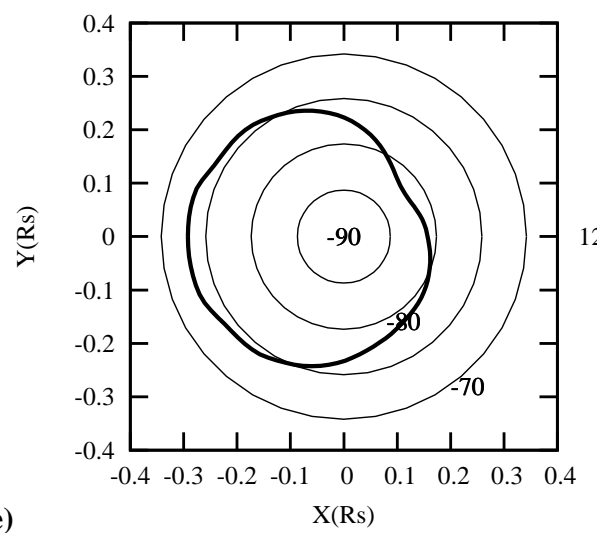

$\operatorname{Bimf}=0.0 ;-0.4 ;-0.4 ; \mathrm{ks}=0.2$

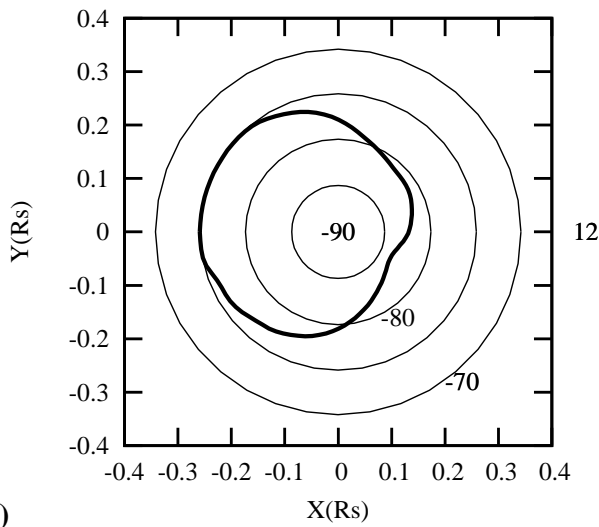

$\operatorname{Bimf}=0.5 ;-2.0 ;-1.4 ; \mathrm{ks}=0.2$

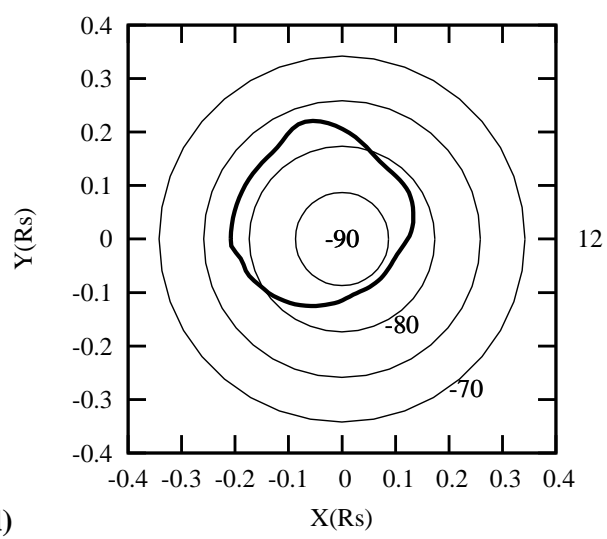

Bimf $=-0.3 ;+0.7 ;+0.7 ; \mathrm{ks}=0.2$

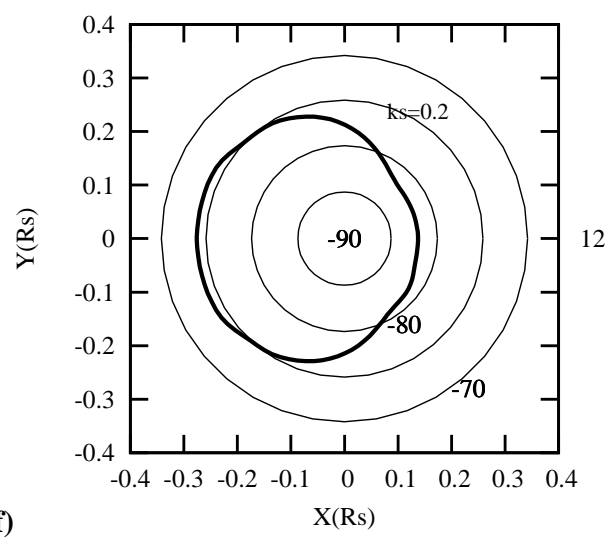

Fig. 5. As for Fig. 4, but showing how the open field area varies with the IMF penetration parameter $k_{S}$. The panels on the left side reproduce those shown in panels (a), (e), and (f) of Fig. 4 with $k_{S}=0.8$, while those shown on the right side have the same IMF vector, but now with $k_{S}=0.2$.

the global magnetospheric magnetic field structure, as can be seen in Fig. 6. This figure shows the magnetospheric field lines for these cases projected onto the $\mathrm{X}-\mathrm{Z}$ plane in the same format as the left-hand panels of Fig. 3. It can be seen that the global size of the modelled region of Saturn's open and closed magnetospheric field lines depends strongly on the
$k_{S}$ value. It should be realised that, although the bounding paraboloid magnetopause is of fixed size in Figs. 3 and 6, considerable volumes in the model "tail" region contain field lines that do not connect with the planet at either end (e.g. Belenkaya, 1998), and are consequently not shown in these figures. 

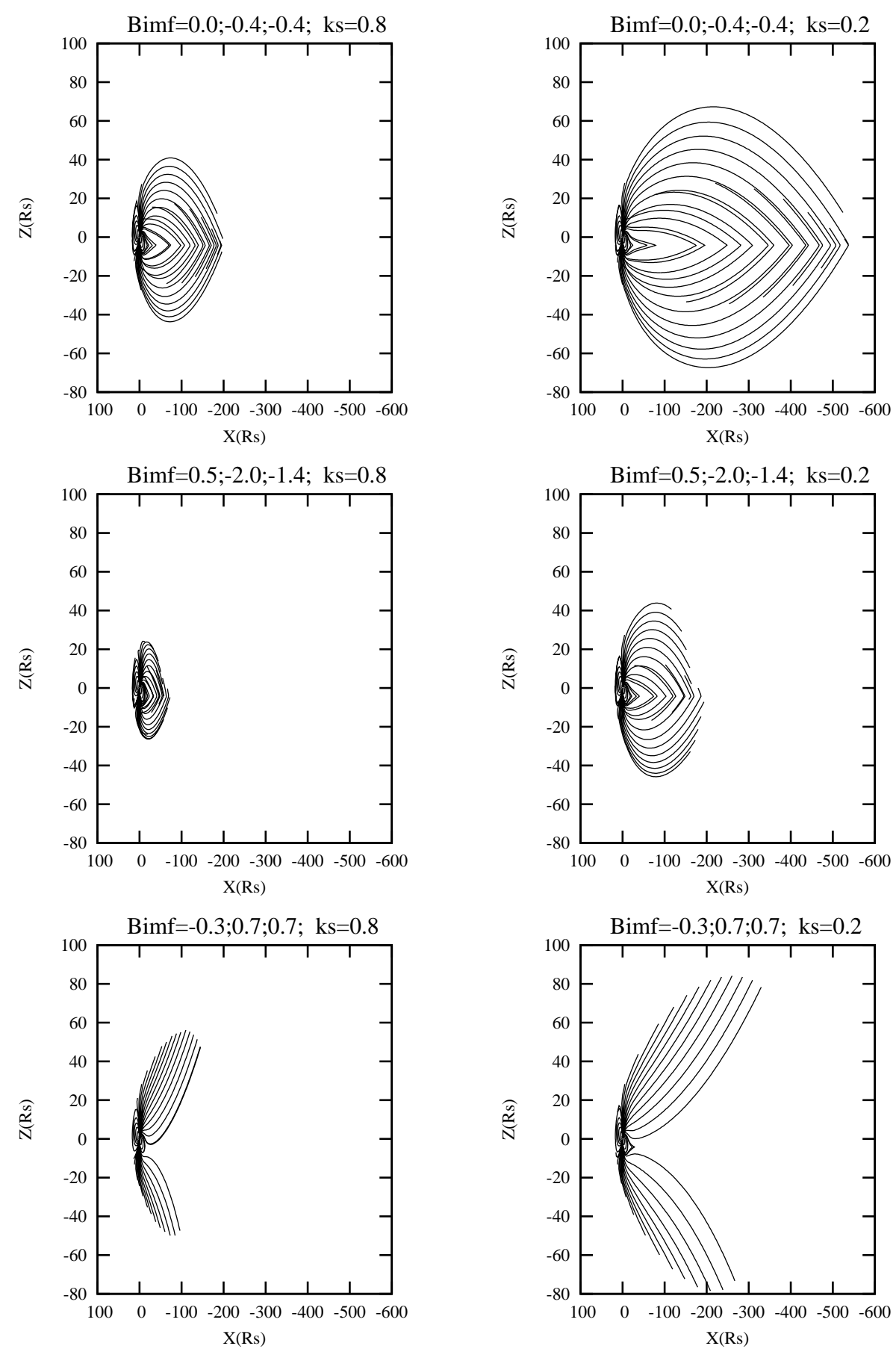

Fig. 6. Plots of magnetospheric field lines projected into the X-Z plane in the same format as the left side of Fig. 3, but now for IMF vectors and penetration coefficients corresponding to the six panels of Fig. 5.

Alexeev et al. (1998) previously showed that even in the case when the model magnetosphere is "closed" (e.g. with $k_{S}=0$ or with no IMF), the model contains long tail lobe magnetic field lines which are directed almost parallel to the equatorial plane, and intersect the distant tail cross section perpendicular to the $\mathrm{x}$-axis. These open field lines may be considered to be formed from previous interactions between the magnetosphere and the interplanetary medium, and may thus be taken to correspond, for example, to the open flux which is present during "deep" rarefaction regions when the IMF is very weak. Alexeev et al. (2006) estimated the open flux in each tail lobe in terms of the 
model parameters as $\Phi_{0}=(\pi / 2)\left(1+2 R_{2} / R_{S S}\right) B_{t} R_{s s}^{2}$ (see also Belenkaya et al., 2006b). This represents the "baseline" open flux that is present for the model for $k_{S}=0$. For the chosen magnetospheric input parameters employed here $\left(R_{s s}=17.5 R_{S}, \quad R_{2}=14 R_{S}, \quad B_{t}=8.7 \mathrm{nT}\right) \quad$ we obtain $\Phi_{0}=7529.5 \mathrm{nT} R_{S}^{2}(27.4 \mathrm{GWb})$. We can readily estimate the ionospheric co-latitude corresponding to this amount of magnetic flux. The dipole magnetic field strength at Saturn's equator is $B_{S 0}=21160 \mathrm{nT}$, so that the magnetic field strength at the pole is roughly $B_{p}=2 B_{S 0}=42320 \mathrm{nT}$. The flux $\Phi_{0}$ can also be written as $B_{p} S_{0}$, where $S_{0}$ is the area of the open field line region in the ionosphere for the "baseline" model, and $S_{0}=\pi \rho^{2}$, where $\rho$ is the radius of this region, and $\rho=R_{S} \sin \theta$, where $\theta$ is the co-latitude of the open-closed field line boundary. Thus, $\Phi_{0}=B_{p} \pi R_{S}^{2} \sin ^{2} \theta$, and correspondingly $\sin \theta=\left(\Phi_{0} /\left(B_{p} \pi R_{S}^{2}\right)\right)^{1 / 2}=\left(7529.5 \mathrm{nT} R_{S}^{2} /\left(42320 \mathrm{nT} \pi R_{S}^{2}\right)\right)^{1 / 2}$ $=0.24$, thus $\theta \sim 14^{\circ}$. This value may be considered to provide a rough estimation of the effective open field line region boundary co-latitude for the "baseline" model with $k_{S}=0$.

It is well known for the case of the Earth that the radius of the region of open field lines depends on the north-south component of the IMF. The experimental evidence is summarized, for example, by Holzworth and Meng (1984) and Bolshakova et al. (1988), while the dependence in numerical models was obtained, for example, by Alexeev et al. (1993) and Belenkaya (1998). For the Earth the open field line region increases for southward IMF, and decreases for northward IMF, which is the opposite for Saturn, due to the opposite sense of the planetary magnetic moment. The situation which most closely represents the "baseline" case in those computed here is Fig. $5 f$ for a weak northward IMF. We see that for this case the boundary is located at $\sim 12.5^{\circ}$, which is in reasonable accord with the rough $\left(14^{\circ}\right)$ estimation above, based on the open tail lobe flux. We also note that Alexeev (2005) gave a relation which determines the size of the polar cap for the "ground state" of the Earth's magnetosphere. Recasting Alexeev's (2005) Eq. (6) for the case of Saturn's magnetosphere, we find $\sin \theta=\left(2.2 R_{S} / 3 R_{S S}\right)^{1 / 2}=0.2$, which gives $\theta=11.8^{\circ}$. The difference of $0.7^{\circ}$ between this value of the polar cap size and that shown in Fig. $5 f$ may be due to the weak northward IMF, or physically to small magnetospheric disturbances. Both factors increase the amount of open flux in the magnetosphere. The same factors may also explain the modestly increased flux in our $k_{S}=0$ "baseline" Saturn model compared with Alexeev's (2005) estimation. We finally note that the "baseline" model should also provide an initial description of magnetic field conditions during "deep" rarefaction regions when the IMF is very weak. However, this is not a matter we explore in detail here, concentrating instead on conditions during solar wind compressions.

\section{Comparison with auroral observations}

We now compare the results shown in Figs. 4 and 5 with the observed auroral distributions shown in Fig. 2, which correspond to the interplanetary data shown in Fig. 1, as indicated by the vertical dashed lines (within the few-hour uncertainties of the latter's timing). As discussed previously by Badman et al. (2005) and Bunce et al. (2006), the first HST observations during the 2004 Cassini campaign, on 8 January, were obtained during a "deep" rarefaction region. The corresponding auroral oval (image a) was highly expanded at $15^{\circ}-20^{\circ}$ co-latitude, with brightenings observed in the premidnight, dawn, and pre-noon sector. By 15 January (image e) the open field line region radius was a little smaller, around $12^{\circ}-18^{\circ}$. After the arrival of the "minor" solar wind compression at Saturn (on 16 January), the radius of the contracted open field line region varied from $5^{\circ}-8^{\circ}$ in the postmidnight and dawn sectors to $\sim 13^{\circ}$ in the post-noon sector, with the brightness decreasing from post-midnight anticlockwise into the pre-midnight hours. During the subsequent "intermediate" rarefaction region, a re-expanded oval was seen on 23 January (image i) with auroral brightenings at dawn and noon. On 26 January, at the onset of the "major" CIR compression, a highly brightened and contracted oval was observed (image k), with bright auroral forms on the dawnside from $\sim 3^{\circ}$ to $\sim 15^{\circ}$ co-latitude. On 30 January, near the end of the "major" compression, bright auroras were still observed except in the pre-midnight sector (image $m$ ), and the oval re-expanded to $12^{\circ}-15^{\circ}$ co-latitude in the pre-dawn region.

We now more directly compare the calculated open field line areas shown in Fig. 5 with the corresponding compression region auroral images shown in Fig. 2. In Fig. 7 panels (a-c) we thus show images (f), (k), and (m), respectively, projected onto a polar grid in which the southern ionosphere is viewed "through" the planet from the north, as in Figs. 4 and 5, with noon now at the bottom of each plot, and dawn to the left. The dotted circles are at intervals of $10^{\circ}$ co-latitude, and the auroral intensities are indicated by the colour scale shown on the right. Smoothed calculated open field boundaries are then over-plotted on the images, where the solid lines correspond to $k_{S}=0.2$ (Figs. $5 \mathrm{~b}, \mathrm{~d}$, and $\mathrm{f}$ in (a), (b), and (c) respectively), and the dashed lines to $k_{S}=0.8$ (similarly Figs. 5a, c, and e). Some important points emerge from these comparisons. First, the size of the calculated area of open flux generally agrees very well with the area encircled by the UV auroras, within the limitations imposed by the continuity of the observed auroral emission in local time. The boundaries for the two $k_{S}$ values are essentially indistinguishable in panels (b) and (c), while in panel (a) the larger area for $k_{S}=0.2$ agrees better with the area poleward of the emissions than does the smaller area for $k_{S}=0.8$. These results thus provide support for the hypothesis made by Badman et al. (2005) that the dark area poleward of the auroral emission corresponds to open field lines. Second, with regard to the detailed 
(a) 2004-01-18 04:40:14

00

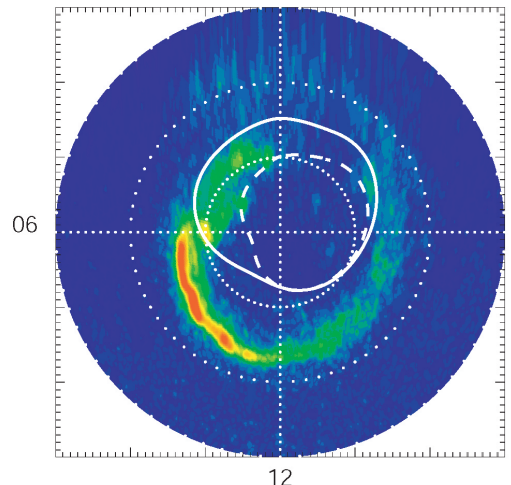

(b) 2004-01-26 19:02:32

00

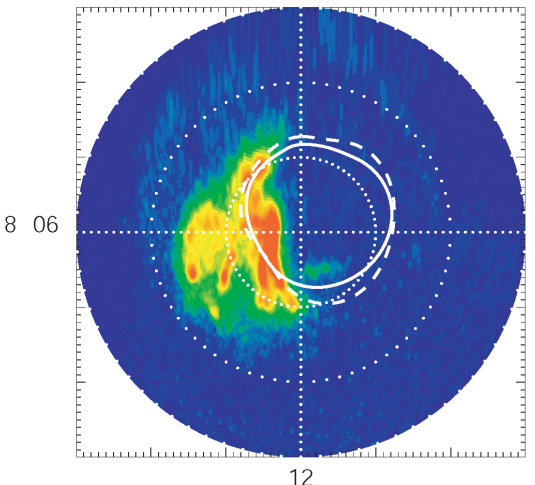

(c) 2004-01-30 19:01:19

00

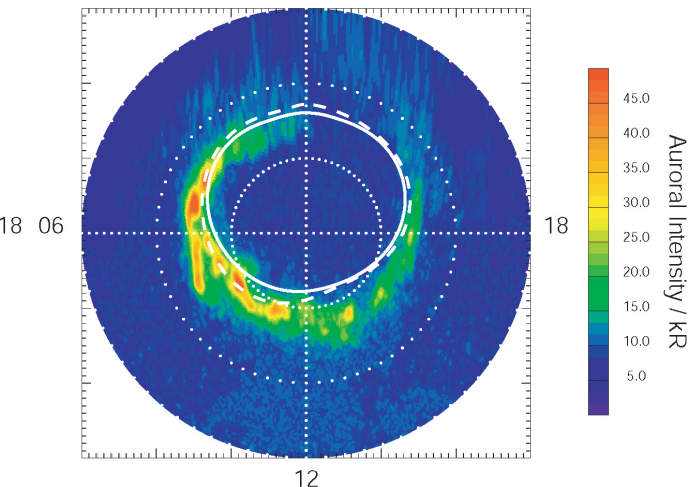

Fig. 7. UV images of Saturn's southern polar aurora obtained during January 2004 are shown projected onto a polar grid, from the pole to $30^{\circ}$ co-latitude, again viewed as though looking "through" the planet onto the southern pole, as in Figs. 4 and 5. However, noon is now at the bottom of each plot and dawn to the left, as indicated. The UV auroral intensity is colour-coded according to the scale shown on the right-hand side of the figure. Panels (a-c) show images (f), (k), and (m) in Fig. 2, respectively, whose HST start times are given at the top of each plot. These projected images are reproduced from Badman et al. (2005). The over-plotted solid and dashed white lines show the smoothed calculated locations of the boundary of open field lines for $k_{S}=0.2$ and 0.8 , respectively. The curves in panel (a) thus correspond to Figs. 5a and b, respectively, those in panel (b) to Figs. 5c and d, and those in panel (c) to Figs. 5e and f.

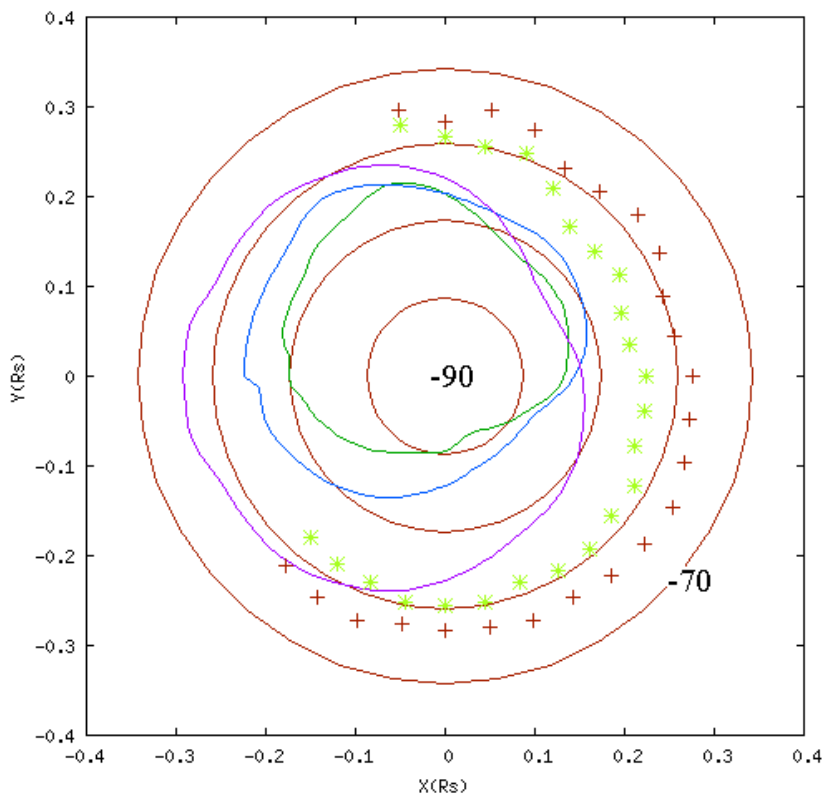

Fig. 8. Comparison of the computed Southern Hemisphere open field regions for $k_{S}=0.8$ shown in panels (a), (c), and (e) of Fig. 5 (and a, e, and f of Fig. 4), shown by the green, blue, and purple lines, respectively, with the median poleward (yellow stars) and equatorward (red crosses) southern auroral boundary locations determined by Badman et al. (2006) (their Fig. 5). The plot format is the same as for Figs. 4 and 5. The red lines show co-latitude circles at $5^{\circ}$ intervals from the southern pole to $-70^{\circ}$. position of the calculated open field region relative to the auroras, it can be seen that this agrees very well with the poleward boundary of the auroras in panel (c) (for which the IMF is northward). In panel (b) (for which the IMF is southward), the bright dawn auroras also lie at and just inside the calculated open field boundary in this local time sector. Finally, in panel (a) (for which the IMF is weakly southward) the calculated boundary for $k_{S}=0.2$ is of a similar size and shape as the poleward boundary of the auroras, as just indicated, but the latter are displaced significantly toward noon, compared, for example, with the similar distribution shown in panel (c). For further discussion of the auroral distributions seen in these images and their relationship to solar wind-magnetosphereionosphere coupling current systems, the reader is referred to the results of Belenkaya et al. (2006a), discussed briefly in the Introduction.

We can also compare these calculated open field boundaries with the typical position of the UV oval determined by Badman et al. (2006). These authors presented an analysis of a selection of twenty-two HST images of Saturn's auroras obtained during 1997-2004, including the images shown here in Fig. 2. In Fig. 8 the yellow stars and red crosses show the median poleward and equatorward boundaries of the aurora determined from these images, respectively, plotted on a polar grid in the same format as Figs. 4 and 5. The local time coverage ranges from the pre-dawn sector to post-dusk via noon. The green, blue, and purple curves then show the calculated open field boundaries for $k_{S}=0.8$ shown in panels (a), (c), and (e) of Fig. 5 (and a, e, and f of Fig. 4), respectively, thus corresponding to the "minor" compression region, the 
initial "major" compression region, and the later "major" compression region, respectively, in the January 2004 data. It can be seen that all of these calculated boundaries lie a few degrees poleward of the median poleward auroral boundary in the noon sector, roughly intermediate between panels (a) and (c) in Fig. 7, while the largest boundary approaches the median oval in the dawn sector.

\section{Conclusions}

In this paper we have investigated the IMF-dependence of the open field line region in Saturn's ionosphere using the paraboloid model of the magnetosphere, combined with IMF data obtained by the Cassini spacecraft during its approach to Saturn in January 2004. We have compared these results with simultaneous images of Saturn's UV auroras obtained by the HST, specifically the bright active emissions observed during interplanetary compression regions when the IMF is strongest. It has been shown that the IMF direction significantly changes the magnetospheric magnetic field structure, together with the area and shape of the region of open field lines. Comparison with related auroral images shows that the calculated area of open field lines is comparable to that enclosed by the auroral oval, in support of previous hypotheses to this effect. In addition, the position of the calculated open field region sometimes agrees in detail with the poleward boundary of the auroral oval, though more typically being displaced by a few degrees of latitude in the tailward direction. We thus conclude on the basis of these results that the solar wind and its magnetic field plays a major role in the generation of Saturn's auroras.

Acknowledgements. Work at the Institute of Nuclear Physics, Moscow State University was supported by INTAS Grant No 0351-3922 and by the RFBR Grants 05-05-64435, 06-05-64508, and 07-05-00529. Work at Leicester was supported by PPARC/STFC grants PPA/G/O/2003/00013 and PP/E000983/1. SWHC was supported by a Royal Society Leverhulme Trust Senior Research Fellowship. The auroral data from which Figs. 2, 7, and 8 have been prepared are courtesy of J. Clarke (Boston University), and J.-C. Gérard and D. Grodent (Université de Liège).

Topical Editor I. A. Daglis thanks J. Nichols and another referee for their help in evaluating this paper.

\section{References}

Alexeev, I. I.: The penetration of interplanetary magnetic and electric fields into the magnetosphere, J. Geomagn. Geoelectr., 38, 1199-1221, 1986.

Alexeev, I. I., Belenkaya, E. S., Bobrovnikov, S. Yu., and Kalegaev, V. V.: Modelling of the electromagnetic field in the interplanetary space and in the Earth's magnetosphere, Space Sci. Rev., 107, 7-26, 2003.

Alexeev, I. I.: What defines the polar cap and auroral oval diameters?, in: The Inner Magnetosphere: Physics and Modeling, edited by: Pulkkinen, T. I., Tsyganenko, N. A., and Friedel, R. H. W., Geophys. Mon. Ser., 155, 257-262, AGU, 2005.

Alexeev, I. I., Belenkaya, E. S., and Sibeck, D. G.: Open field lines in the closed model of the magnetosphere (in Russian), Geomagn. Aeron., 38, 9-18, 1998.

Alexeev, I. I., Belenkaya, E. S., Kalegaev, V. V., and Lutov, Yu. G.: Electric fields and field-aligned current generation in the magnetosphere, J. Geophys. Res., 98, 4041-4051, 1993.

Alexeev, I. I., Kalegaev, V. V., Belenkaya, E. S., Bobrovnikov, S. Y., Bunce, E. J., Cowley, S. W. H., and Nichols, J. D.: A global magnetic model of Saturn's magnetosphere, and a comparison with Cassini SOI data, Geophys. Res. Lett., 33, L08101, doi:10.1029/2006GL025896, 2006.

Badman, S. V., Bunce, E. J., Clarke, J. T., Cowley, S. W. H., Gérard, J.-C., Grodent, D., and Milan, S. E.: Open flux estimates in Saturn's magnetosphere during the January 2004 CassiniHST campaign, and implications for reconnection rates, J. Geophys. Res., 110, A11216, doi:10.1029/2005JA011240, 2005.

Badman, S. V., Cowley, S. W. H., Gérard, J.-C., and Grodent, D.: A statistical analysis of the location and width of Saturn's southern auroras, Ann. Geophys., 24, 3533-3545, 2006, http://www.ann-geophys.net/24/3533/2006/.

Belenkaya, E. S.: High-latitude ionospheric convection patterns dependent on the variable IMF orientation, J. Atmos. Solar-Terr. Phys., 60/13, 1343-1354, 1998.

Belenkaya, E. S.: The jovian magnetospheric magnetic and electric fields: Effects of the interplanetary magnetic field, Planet. Space Sci., 52, 499-511, 2004.

Belenkaya, E. S., Cowley, S .W. H., and Alexeev, I. I.: Saturn's aurora in the January 2004 events, Ann. Geophys., 24, 16491663, 2006a.

Belenkaya, E. S., Alexeev, I. I., Kalegaev, V. V., and Blokhina, M. S.: Definition of Saturn's magnetospheric model parameters for the Pioneer 11 flyby, Ann. Geophys., 24, 1145-1156, $2006 \mathrm{~b}$.

Bolshakova, O. V., Kleimenova, N. G., and Kurazhkovskaya, N. A.: Polar cap dynamics on the observations of the long period geomagnetic pulsations (in Russian), Geomagn. Aeron., 28, 661665, 1988.

Bunce, E. J., Cowley, S. W. H., Jackman, C. M., Clarke, J. T., Crary, F. J., and Dougherty, M. K.: Cassini observations of the interplanetary medium upstream of Saturn and their relation to Hubble Space Telescope aurora data, Adv. Space Res., 38, 806$814,2006$.

Clarke, J. T., Gérard, J.-G., Grodent, D., Wannawichian, S., Gustin, J., Connerney, J., Crary, F., Dougherty, M., Kurth, W., Cowley, S. W. H., Bunce, E. J., Hill, T., and Kim, J.: Morphological differences between Saturn's ultraviolet aurorae and those of Earth and Jupiter, Nature, 433, 717-719, 2005.

Cowley, S. W. H. and Bunce, E. J.: Origin of the main auroral oval in Jupiter's coupled magnetosphere-ionosphere system, Planet. Space Sci., 49, 1067-1088, 2001

Cowley, S. W. H. and Bunce, E. J.: Corotation driven magnetosphere-ionosphere coupling currents in Saturn's magnetosphere and their relation to the auroras, Ann. Geophys., 21, 1691-1707, 2003, http://www.ann-geophys.net/21/1691/2003/.

Cowley, S. W. H., Bunce, E. J., and Prangé, R.: Saturn's polar ionospheric flows and their relation to the main auroral oval, Ann Geophys., 22, 1379-1394, 2004a. 
Cowley, S. W. H., Bunce, E. J., and O'Rourke, J. M.: A simple quantitative model of plasma flows and currents in Saturn's polar ionosphere, J. Geophys. Res., 109, A05212, doi:10.1029/2003JA010375, 2004b.

Crary, F. J., Clark, J. T., Dougherty, M. K., Hanlon, P. G., Hansen, K. C., Steinberg, J. T., Barrachlough, B. L., Coates, A. J., Gérard, J.-C., Grodent, D., Kurth, W. S., Mitchell, D. G., Rymer, A. M., and Young, D. T.: Solar wind dynamic pressure and electric field as the main factors controlling Saturn's auroras, Nature, 433, 720-722, 2005.

Dougherty, M. K., Kellock, S., Southwood, D. J., Balogh, A., Smith, E. J., Tsurutani, B. T., Gerlach, B., Glassmeier, K.-H., Gleim, F., Russell, C. T., Erdos, G., Neubauer, F. M., and Cowley, S. W. H.: The Cassini magnetic field investigation, Space Sci. Rev., 114, 331-383, 2004.

Hill, T. W.: The jovian auroral oval, J. Geophys. Res., 106, 81018107, 2001.

Holzworth, R. H. and Meng, C.-I.: Auroral boundary variations and the interplanetary magnetic field, Planet. Space Sci., 32, 25-29, 1984.

Gérard, J.-C., Dols, V., Grodent, D., Waite, J. H., Gladstone, G. R., and Prangé, R.: Simultaneous observations of the saturnian aurora and polar haze with the HST/FOC, Geophys. Res. Lett., 22, 2685-2688, 1995.
Gérard, J.-C., Grodent, D., Gustin, J., and Saglam, A.: Characteristics of Saturn's FUV aurora observed with the Space Telescope Imaging Spectrograph, J. Geophys. Res., 109, A09207, doi:10.1029/2004JA010513, 2004.

Jackman, C. M., Achilleos, N., Bunce, E. J., Cowley, S. W. H., Dougherty, M. K., Jones, G. H., and Milan, S. E.: Interplanetary magnetic field at $\sim 9 \mathrm{AU}$ during the declining phase of the solar cycle and its implications for Saturn's magnetospheric dynamics, J. Geophys. Res., 109, A11203, doi:10.1029/2004JA010614, 2004.

Sandel, B. R. and Broadfoot, A. L.: Morphology of Saturn's aurora, Nature, 292, 679-682, 1981.

Trauger, J. T., Clarke, J. T., Ballester, G. E., Evans, R. W., Burrows, C. J., Crisp, D., Gallagher III, J. S., Griffiths, R. E., Hester, J. J., Hoessel, J. G., Holtzman, J. A., Krist, J. E., Mould, J. R., Sahai, R., Scowen, P. A., Stapelfeldt, K. R., and Watson, A. M.: Saturn's hydrogen aurora: Wide field and planetary camera 2 imaging from the Hubble Space Telescope, J. Geophys. Res., 103, 20 237-20 244, 1998.

Tsyganenko, N. A.: A model of the near magnetosphere with a dawn-dusk asymmetry. Mathematical structure, J. Geophys. Res., 107, A8, doi:10.1029/2001JA000219, 2002.

Young, D. T., Berthelier, J. J., Blanc, M., et al.: Cassini Plasma Spectrometer investigation, Space Sci. Rev., 114, 1-112, 2004. 